\title{
Originals
}

\section{An Epidemiological Study of Childhood Diabetes Affecting Two or More Siblings}

\author{
D. R. Gamble \\ The Public Health Laboratory, West Park Hospital, Epsom, England
}

Summary. The incidence, prevalence, and ages and dates of onset of diabetes, were studied in 184 families with 2 or more affected children. Results suggested that the siblings of children who developed diabetes before the age of 16 years were 26 times more likely to develop diabetes than other children. Of all siblings surveyed it was estimated that $5.6 \%$ became diabetic by the age of 16 . The distribution of ages at onset in these siblings was similar to that in the general population, and within sibships, age at onset appeared to be independently determined. An interval of less than a year between the dates of onset in siblings occurred with more than twice the expected frequency, and in most the interval was less than 6 months. These results suggest that age at onset is determined by non-genetic factors and that, in at least some cases, aetiological environmental factors may lead to the development of diabetes within a period of a few months.

Key words: Insulin-dependent diabetes, sibships, childhood diabetes, incidence, extrinsic factors, contemporaneous onset.

The pathogenesis of insulin-dependent diabetes involves both genetic and environmental factors [1] and study of the disease in families can provide information about both aspects. Many family studies have been undertaken [2], but information about childhood diabetes in sibships has been limited. The present report describes a preliminary epidemiological study of 184 families in which diabetes affected two or more siblings before the age of sixteen.

\section{Methods}

Complete details of the calculations are available on request from the author: the general principles of the methods employed are described below.
In 1972, the British Diabetic Association started a Register to which notification was invited of all new cases of diabetes in children aged $0-15$ years, in Great Britain and Ireland [3]. Diagnostik criteria were not defined and the type of diabetes was not restricted, but $99 \%$ of notified patients were treated with insulin. In an epidemiological study based on this register, details were obtained by questionnaire of the numbers and ages of siblings of probands, and the dates of onset of diabetes affecting any siblings, including those affected before 1972 .

The distribution of age at onset of diabetes in 326 siblings was compared with the distribution in diabetic children in general based on ages of onset of 3538 cases notified in 1973-5 [4].

Observed differences in age at onset in 179 sibling pairs were compared with the calculated distribution for random pairs of unrelated diabetic children. For this calculation, probabilities of onset at each age from 0 to 15 years were derived from the relative frequencies at these ages in the general population [4]. Thence, the probabilities of the possible permutations of ages at onset in sibships were calculated. Each of these permutations comprised two ages at onset differing by 0 to 15 years; by grouping permutations with the same age difference, and summating their corresponding probabilities, the probabilities of differences in age at onset of 0 to 15 years were obtained.

Observed differences between dates of onset (discordance intervals) in 179 sibling pairs were compared with the calculated distribution of discordance intervals in pairs of unrelated diabetic children. The difference in age of each reported sibling pair was known, and for a given difference in age the discordance interval depends solely on the difference in age at onset. For each sibling pair, the probabilities of different discordant intervals were therefore calculated for each possible permutation of their ages at onset. Thence, probabilities for discordance intervals of $0-15$ years were summated.

To assess the incidence of diabetes in siblings, the number of siblings at risk was estimated. The cumulative frequencies of notified diabetic children at ages $0-15$ years was obtained by cumulation of previously determined age specific annual frequencies [4]; mortality at these ages is low and was ignored. Questionnaires received in respect of 4868 of the 6600 notified patients contained details of the number and ages of patients' siblings, and the age structure of sibships that include a diabetic child was estimated from these data. The numbers and ages of siblings in the families of 100 probands of each age from 1 to 15 years, and the families of the 16 notified probands aged less than a year, were used to compile this estimate which, when adjusted to the cumulative frequencies of notified patients aged $0-15$ years, provided an estimate of the number of siblings at risk (Table 1). Age-specific incidence in siblings was determined by dividing the average numbers ascertained per year by the numbers at risk; prevalence was obtained by cumulating incidence. 


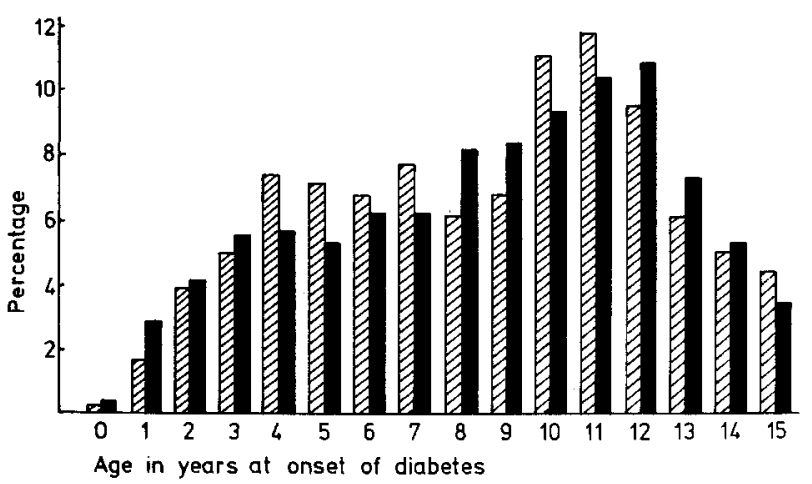

Fig. 1. Distribution of age at onset of diabetes in 326 children in diabetic sibships (stripes) and 3538 diabetic children in the population as a whole (black). $\chi_{15}^{2}=12.01(0.60<\mathrm{P}<0.70) .26$ sibling pairs were excluded from this analysis because the age of the index sibling at onset was not available

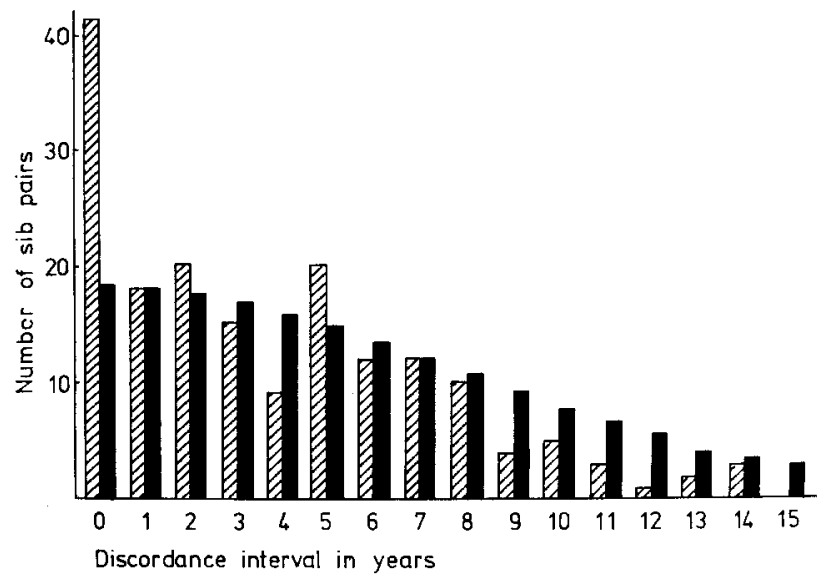

Fig. 3. Distribution of differences between dates of onset in 177 diabetic sibling pairs. Observed (striped) and expected (black), $\chi_{12}^{2}$ $=43.67(\mathrm{P}<0.001)$. Two pairs in which the discordance interval exceeded 15 years are not shown in the figure. The expected distribution was derived from the calculated distribution of discordance intervals expected in 177 pairs of unrelated diabetic children with age differences within pairs matching those of the 179 diabetic sibling pairs (see text)

Of the 11 pairs of diabetic twins in the study, 1 was dizygotic, 3 monozygotic, and 7 of unknown zygosity. The dizygotic pair were included for the calculation of incidence in siblings but the rest were omitted (see results).

Patients who develop diabetes after the age of 15 years may have siblings aged $0-15$ years but, as the frequency of diabetes after the age of 15 years is poorly documented, patients aged 16 years and over were omitted both from the numbers of ascertained cases in sibships, and from the estimation of the numbers of siblings at risk.

All tests of statistical significance of results employed the conventional Chi-square test.

\section{Results}

In an analysis of data available at September 1978, completed questionnaires had been received from $4868(74 \%)$ of 6,600 patients. Diabetes affecting a

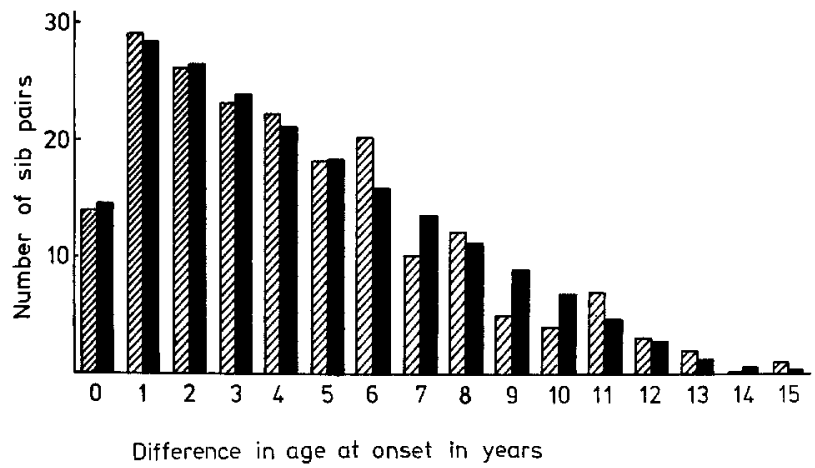

Fig. 2. Distribution of differences in age at onset of diabetes in 179 sibling pairs. Observed (striped) and expected (black). $\chi_{12}^{2}=$ $6.603(0.80<\mathrm{P}<0.90)$. The expected distribution was derived from the calculated differences in age at onset expected in random pairs of unrelated diabetic children (see methods)

sibling of a proband was reported in 184 families. There were 378 affected children including 2 diabetic children in 175 families, 3 in 8 families, and 4 in 1 family. In 26 families the age of the index case was not obtained but ages and dates of onset were available for 179 sibling pairs.

The age distribution of age at onset of diabetes in these families (Fig. 1) was similar to that in the population as a whole $(0.60<\mathrm{P}<0.70)$.

If the age at onset were mainly determined by genetic factors, onset in siblings would tend to occur at about the same age. The distribution of differences in age at onset in sib pairs was therefore compared with the distribution expected if ages at onset in sibling pairs were independently determined (Fig. 2). There was a good fit between observed and expected distribution $(0.80<\mathrm{P}<0.90)$ indicating independence of ages at onset in sibships.

In a few sibships, the almost simultaneous onset of diabetes suggested the influence of environmental factors. Thus, diabetes affecting two siblings within 4 weeks was reported in 7 families, in three of which, onset occurred in the same week. Simultaneous onset in siblings may also occur by chance and observed discordance intervals were therefore compared with the distribution expected if the time of onset were independently determined in each sibling (Fig. 3). There was a striking departure from the expected distribution with more than twice the expected number of sibships having a discordance interval of less than 1 year $(\mathrm{P}<0.001)$. Moreover, of the 41 sibling pairs in this category, $32(78 \%)$ had discordance intervals of less than 6 months, nearly 4 times the expected frequency.

The average numbers of diabetic siblings reported per year were related to the estimated numbers at risk to provide an estimate of the incidence in siblings (Table 1). Although ascertainment was incomplete, in the calculation of incidence the deficit affected 
Table 1. Diabetes affecting 2 or more siblings at ages $0-15$ years. Incidence and Prevalence estimate

\begin{tabular}{|c|c|c|c|c|c|}
\hline \multirow[t]{2}{*}{ Age at onset (years) } & \multirow{2}{*}{$\begin{array}{l}\text { 1st diabetic sibling } \\
\text { No. reported }\end{array}$} & \multicolumn{4}{|c|}{ 2nd and subsequent diabetic siblings } \\
\hline & & No. reported ${ }^{\mathrm{b}}$ & Average no. per year ${ }^{\mathrm{c}}$ & No. of siblings at risk ${ }^{d}$ & $\begin{array}{l}\text { Incidence peryear } \\
\%\end{array}$ \\
\hline 0 & 1 & - & 0 & 199 & 0.0 \\
\hline 1 & 5 & 1 & 0.24 & 207 & 0.12 \\
\hline 2 & 10 & 1 & 0.24 & 397 & 0.06 \\
\hline 3 & 13 & 3 & 0.73 & 309 & 0.24 \\
\hline 4 & 11 & 10 & 2.43 & 507 & 0.48 \\
\hline 5 & 14 & 8 & 1.94 & 592 & 0.33 \\
\hline 6 & 9 & 13 & 3.16 & 527 & 0.60 \\
\hline 7 & 10 & 12 & 2.92 & 905 & 0.32 \\
\hline 8 & 10 & 10 & 2.43 & 781 & 0.31 \\
\hline 9 & 9 & 15 & 3.64 & 1001 & 0.36 \\
\hline 10 & 15 & 24 & 5.83 & 950 & 0.61 \\
\hline 11 & 11 & 27 & 6.56 & 1116 & 0.59 \\
\hline 12 & 11 & 24 & 5.83 & 890 & 0.66 \\
\hline 13 & 8 & 15 & 3.64 & 999 & 0.37 \\
\hline 14 & 5 & 13 & 3.14 & 980 & 0.32 \\
\hline 15 & 6 & 8 & 1.94 & 740 & 0.26 \\
\hline Not known & 26 & & & & \\
\hline $0-15$ & 174 & 184 & 44.67 & 11.100 & 5.63 \\
\hline
\end{tabular}

a Of the 184 patients reported, 10 occurring in twinships were excluded (see results)

b There were 194 affected siblings in the 184 families, but 10 occurring in twinships were excluded (see results)

c 4868 questionnaires were analysed, and from 1973 to 1976,1183 notifications p. a. were received. The average number of diabetic siblings

ascertained therefore $=\frac{\text { number reported } \times 1183}{4868}$

d See methods

both the numerator (diabetic siblings reported) and the denominator (number at risk) to approximately the same extent, since both were based on the frequency of notified cases. Although based on small numbers variation of incidence in siblings with age showed the expected pattern of a major peak at 11 years and a shoulder at about 4 years. The average incidence for ages $0-15$ years was $0.36 \%$ and the cumulative incidence from $0-15$ years indicated a prevalence of $5.6 \%$ by the age of 16 years, assuming negligible mortality.

Among the 6,600 notified patients there should be 26 monozygotic twinships $(1: 250)$, and 80 dizygotic twinships $(1: 80)$. Since the prevalence of diabetes in identical twins of juvenile diabetics is about $50 \%$ [1], about 13 pairs of diabetic monozygotic twins would be expected but, in view of the low incidence of childhood diabetes in siblings of diabetics $(0.36 \%$ per annum), few if any concordant diabetic dizygotic twinships would be expected. In fact, the diabetic sibships ascertained included 11 pairs of twins - 3 monozygotic, 1 dizygotic, and 7 of uncertain zygosity which were probably monozygotic. The high incidence of diabetes in monozygotic twins would inflate the estimate of incidence of diabetes in siblings, and for this calculation the dizygotic twinship was therefore included but the other 10 twinships were omitted.

\section{Discussion}

The characteristic peaks in the age at onset of insulin dependent diabetes [5] could reflect different levels of genetic susceptibility, but the independence of ages at onset in diabetic sibships suggests that these peaks are more probably due to non-genetic factors. The similarity of the age incidence in siblings to that in the general population, implies that the increased susceptibility of siblings does not result in an earlier age of onset. The age incidence may therefore reflect an increased likelihood of contact with extrinsic aetiological agents at particular ages, or non-genetic intrinsic factors, such as hormonal or growth changes at certain ages, may determine the outcome of contact with extrinsic factors.

The significant excess of short discordance intervals may reflect environmental influence. Selective reporting of twinships and sibships with contemporaneous onset probably contributed to this excess, but it is unlikely to be a major factor. The level of ascertainment of concordant twinships was apparently high, but onset occurred within 6 months in only one of the 11 twinships. Therefore, if the 4-fold excess of sibships with a discordance interval of less than 6 months were due to under-notification of other sibships, it would imply the unlikely supposition that there was a 4-fold difference in the level of 
ascertainment in the two groups, and that the transition occurred abruptly at a discordance interval of 6 months (Fig. 3). It would also imply that ascertainment of cases of childhood diabetes, other than those occurring in sibships, was less than $25 \%$. If this were so, notifications to the register [3] would indicate an incidence in the UK in excess of 30/100,000 per annum, an improbably high incidence compared with that in other Western countries $[6,7]$.

Diabetes in children may prompt investigation of sibs and expedite diagnosis of diabetes, but the analysis was based on dates of onset of clinical symptoms, rather than on dates of diagnosis. However, it is possible that the recognition of earlier and milder symptoms of diabetes in a sibling might in some cases reduce the discordance period, but probably by not more than a few months. A discordance interval of less than 6 months in 32/173 sib pairs suggests that in many of these sibships, diabetes occurred within a short period after exposure to an environmental aetiological agent and in at least one of these sibships diabetes followed mumps. If a diabetogenic process is triggered simultaneously in sibships, the discordance interval would reflect variation in the latent period between initiating event and onset. Discordance intervals in sibships with contemporaneous onset therefore provide an estimate of the variability of the latent period, and the results suggest a range of 6-12 months, or within $\pm 3-6$ months of the median latent period. The longer the latent period, the greater its variability is likely to be, and the narrow range indicated suggests that, in these patients, it is unlikely to be much more than a year. It may be less.

Published incidence data for diabetes in children are limited and conflicting and there may be racial, national and socioeconomic $[6,7,8]$ variations. Perhaps the best data for a European country are from Copenhagen [7], where ascertainment was thought to be virtually $100 \%$, showing an incidence in the 0-14 year age group of $13.7 / 100,000$ per annum. This incidence would imply a prevalence at age 16 of $0.22 \%$. Compared with the estimated prevalence of diabetes in siblings of $5.6 \%$ by the age of 16 years this suggests that siblings of diabetics are 26 times more likely to develop diabetes than other children. This is higher than the estimate of Simpson [9] who found a 15 -fold increased incidence of diabetes in $0-19$ year old siblings of young onset diabetes. The reason for the discrepancy is uncertain but the present estimate of the incidence in siblings may be inflated by selective reporting or the incidence in the population may be higher than estimates suggest.

Two ways in which the aetiology of insulin dependent diabetes might be elucidated are prospective studies, in which non-diabetic siblings of diabetics are followed with a view to identifying the triggering events at or before onset of diabetes, and retrospective studies, in which extrinsic aetiological factors are sought after onset of diabetes. Our results suggest that the incidence of diabetes in siblings of juvenile diabetics is only about 1 in 280 per annum, and that coincidence of onset of diabetes with an extrinsic triggering event is a very rare occurrence. Either approach therefore presents a daunting prospect, but there is probably no easy solution, and it is both remarkable and encouraging that, in two recent applications of the retrospective approach, an apparently causative virus has been identified $[10,11]$.

Acknowledgements. Thanks are due to the British Diabetic Association for financial support, to the many physicians who supplied details about their patients, and in particular to Dr. Arnold Bloom of the Whittington Hospital, for initiating the British Diabetic Association Register of Diabetes in Children on which this investigation was based.

\section{References}

1. Tattersall RB, Pyke DA (1972) Diabetes in identical twins. Lancet II: 1120-1125

2. Simpson NE (1976) The genetics of diabetes mellitus - A review of family data. In: Creutzfeldt W, Kobberling J, Neel JV (eds). The genetics of diabetes mellitus. Springer, Berlin Heidelberg New York, p 12

3. Bloom A, Hayes TM, Gamble DR (1975) Register of newly diagnosed diabetic children. Br Med J III: 580-583

4. Gamble DR (1979) Seed and soil - infection and susceptibility. In: Keen J, Pickup JC, Tawalker CV (eds) Epidemiology of diabetes and its vascular complications. International Diabetes Federation, pp 15-18

5. Gamble DR (1976) Viruses and diabetes: An overview with special reference to epidemiological studies. In: Bajaj JS (ed). Proceedings of IX Congress of the International Diabetes Federation. Exerpta Medica, Amsterdam Oxford 1977, p 285-293

6. Jarrett RJ (1979) The epidemiology of young-onset diabetes. In: Keen H, Pickup JC, Tawalkar CV (eds). Epidemiology of diabetes and its vascular complications. International Diabetes Federation, $\mathrm{p}$ 19-21

7. Christau B, Kromann H, Andersen OO, Christy M, Buschard K, Arrung K, Kristensen H, Peitersen B, Steinrud J, Nerup J (1977) Incidence, seasonal and geographical patterns of juvenile onset insulin-dependent diabetes mellitus in Denmark. Diabetologia 13: 281-285

8. Pyke DA (1969) The geography of diabetes. Postgrad Med J 45: 796-801

9. Simpson NE (1968) Diabetes in the families of diabetics. Can Med Assoc J 98: 427-432

10. Gladisch R, Hofmann W, Waldherr R (1976) Myokarditis und Insulitis nach Coxsackie-Virus-Infekt. Z Kardiol 65: 837-849

11. Yoon JW, Austin M, Onodera T, Notkins AL (1979) Virusinduced diabetes mellitus. Isolation of a virus from the pancreas of a child with diabetic ketoacidosis. N Engl J Med 300: 1173-1179

Received: September 3, 1979, and in revised form: May 14, 1980

D. R. Gamble

Public Health Laboratory

West Park Hospital

Epsom, Surrey KT19 6PB, England 